\title{
Upfront Neck Dissection in Chemoradiotherapy for p16-Negative Oropharyngeal Cancer with Neck Metastases: A Retrospective study
}

\author{
Wei Chen Fang ${ }^{1}$, Tsung Lun Lee ${ }^{2}$, Shyh-Kuan Tai ${ }^{1}$, Chia-Fan Chang ${ }^{1}$, Yen-Bin Hsu ${ }^{1}$, \\ Pen-Yuan $\mathrm{Chu}^{1}$, Yi-Fen Wang ${ }^{1}$, Muh-Hwa Yang ${ }^{2}$, Peter Mu-Hsin Chang ${ }^{1}$, and Ling-Wei \\ Wang $^{1}$ \\ ${ }^{1}$ Taipei Veterans General Hospital \\ ${ }^{2}$ National Yang-Ming University
}

March 21, 2021

\begin{abstract}
Objective: To determine the role of upfront neck dissection (ND) in patient survival and regional control of p16-negative oropharyngeal squamous cell carcinoma (OPSCC) with neck metastases. Design: Retrospective study. Participants: Patients with p16-negative OPSCC with neck metastases, diagnosed between January 1, 2011 and December 31, 2017, and treated with upfront ND followed by chemoradiotherapy (ND + CCRT) or primary chemoradiotherapy (CCRT). Main outcome measures: Recurrence and survival rates were analysed using the Kaplan-Meier method. Results: Data of 67 patients with p16-negative $\mathrm{cN}+$ OPSCC were analysed. Of them, 23 (34.3\%) received ND + CCRT and 44 (65.7\%) received primary CCRT. At a median follow-up of 37.9 months, the 3-year neck recurrence rate was significantly lower in the ND + CCRT group than in the CCRT group ( $0 \%$ vs. $19 \%, \mathrm{p}=0.031)$. This trend was more obvious in patients with neck metastases [?] $3 \mathrm{~cm}(0 \%$ vs. $32.1 \%, \mathrm{p}=0.045)$. Survival outcomes were comparable between the groups; notably, the ND + CCRT group received a significantly lower dose of radiotherapy (3-year disease-specific survival: $77.3 \%$ and $75.3 \%, \mathrm{p}=0.968$, respectively; 3-year disease-free survival: $77.3 \%$ and $70.1 \%, \mathrm{p}=0.457$, respectively; 3 -year overall survival: $62 \%$ and $61.8 \%, \mathrm{p}=0.771$, respectively between the ND $+\mathrm{CCRT}$ and CCRT groups). Conclusion: Upfront ND was significantly beneficial for regional control and resulted in comparable oncological outcomes with a significantly reduced radiation dose. These results findings can help guide the development of a standardised treatment plan for p16-negative OPSCC. Additional prospective studies with larger sample sizes are warranted.
\end{abstract}

\section{Abstract}

Objective: To determine the role of upfront neck dissection (ND) in patient survival and regional control of p16-negative oropharyngeal squamous cell carcinoma (OPSCC) with neck metastases.

Design: Retrospective study.

Participants: Patients with p16-negative OPSCC with neck metastases, diagnosed between January 1, 2011 and December 31, 2017, and treated with upfront ND followed by chemoradiotherapy (ND + CCRT) or primary chemoradiotherapy (CCRT).

Main outcome measures: Recurrence and survival rates were analysed using the Kaplan-Meier method.

Results: Data of 67 patients with p16-negative cN+ OPSCC were analysed. Of them, $23(34.3 \%)$ received $\mathrm{ND}+\mathrm{CCRT}$ and $44(65.7 \%)$ received primary CCRT. At a median follow-up of 37.9 months, the 3-year neck recurrence rate was significantly lower in the ND + CCRT group than in the CCRT group (0\% vs. $19 \%, p=0.031)$. This trend was more obvious in patients with neck metastases $[?] 3 \mathrm{~cm}(0 \%$ vs. $32.1 \%, p$ 
$=0.045)$. Survival outcomes were comparable between the groups; notably, the ND + CCRT group received a significantly lower dose of radiotherapy (3-year disease-specific survival: $77.3 \%$ and $75.3 \%, p=0.968$, respectively; 3-year disease-free survival: $77.3 \%$ and $70.1 \%, p=0.457$, respectively; 3 -year overall survival: $62 \%$ and $61.8 \%, p=0.771$, respectively between the ND + CCRT and CCRT groups).

Conclusion: Upfront ND was significantly beneficial for regional control and resulted in comparable oncological outcomes with a significantly reduced radiation dose. These results findings can help guide the development of a standardised treatment plan for p16-negative OPSCC. Additional prospective studies with larger sample sizes are warranted.

Keywords: Head and Neck Cancer, Oropharyngeal Neoplasms, Neck Dissection, Chemoradiotherapy

\section{Key Points}

.p16-negative oropharyngeal squamous cell carcinoma was more resistant to radiotherapy than p16-positive lesions.

.p16-negative lesions with large neck metastases [?]3 $\mathrm{cm}$ had poorer oncological outcomes.

.Upfront neck dissection followed by chemoradiotherapy for p16-negative oropharyngeal squamous cell carcinoma improved regional control.

.Compared with primary chemoradiotherapy, an upfront neck dissection followed by chemoradiotherapy can achieve a comparable oncological outcome with a significantly reduced dose of radiotherapy.

.The benefits of upfront neck dissection might be more obvious in patients with large neck metastases.

\section{Introduction}

In oropharyngeal squamous cell carcinoma (OPSCC), p16 is a surrogate marker for the presence of human papillomavirus (HPV), and the prevalence of p16 positivity varies in different countries and areas. In some European and Asian countries, most patients with OPSCC are negative for $\mathrm{p} 16^{(1,2)}$, whereas the opposite is true in North America. ${ }^{(3)}$ However, the prevalence of p16-positive OPSCC might change with time. The United States Food and Drug Administration has recently approved the HPV vaccine for oropharyngeal cancer prevention. Moreover, the universal HPV immunisation programme might help reduce the incidence of p16-positive OPSCC. Thus, improving our understanding of the treatment of p16-negative OPSCC is essential.

The primary difference between p16-positive and p16-negative OPSCC is the tumour's response to chemoradiotherapy; p16-negative OPSCC is generally a poor responder to chemoradiotherapy, which adversely affects oncologic outcomes. ${ }^{(4)}$ Intensified multimodality treatment can enable better management of p16-negative OPSCC.

In addition to chemoradiotherapy, surgical intervention has been advocated in recent years to treat OPSCC. Published studies have mainly focused on the role of surgery for p16-positive OPSCC. ${ }^{(5-9)}$ However, the role of surgical intervention for p16-negative OPSCC, such as neck dissection (ND), remains unexplored. In this study, we investigated the role of ND in patients with p16-negative OPSCC.

\section{Objectives:}

To determine the role of upfront neck dissection (ND) in patient survival and regional control of p16-negative oropharyngeal squamous cell carcinoma (OPSCC) with neck metastases.

To investigate whether upfront ND reduces the postoperative radiation dose and consequent late radiation toxicity.

Design: Retrospective review of patient medical records.

Setting: Tertiary referral head and neck centre. 


\section{Participants:}

Records of 273 patients with OPSCC treated with curative intent between January 1, 2011 and December 31, 2017, were reviewed. Patients who did not complete the entire course of treatment or were not followed up for $>3$ months were excluded. Of 133 patients with p16-negative malignancy, 110 had clinical neck metastases. Patients with simultaneous or prior head and neck malignancy $(n=2)$ or distant metastasis $(\mathrm{n}=41)$ at diagnosis were also excluded. Overall, data of 67 patients with $\mathrm{p} 16$-negative $\mathrm{cN}+$ OPSCC were analysed. The mean follow-up duration was 37.9 months (5-104 months). Clinicopathological data and tumour characteristics were recorded from the hospital registries.

The study design was approved by the hospital's institutional research ethics committee, and the requirement for informed consent from the patients was waived.

\section{Methods:}

All patients underwent standard pre-treatment evaluations for staging. Tumour stages were determined by a Tumor Board using the 7th edition of the American Joint Committee on Cancer/Union for International Cancer Control TNM staging classification. The largest diameters of lymph nodes were measured in the long axis at the meeting and recorded as the maximal nodal size in this review.

Curative treatment was determined according to a shared decision-making approach with the patients, their family, and attending physicians. We explained the current tumour stage and possible treatment options. Then, a treatment plan was initiated based on the willingness of the patients, i.e., either primary concurrent chemoradiotherapy (CCRT) or upfront surgery including ND followed by chemoradiotherapy.

Standard radiotherapy performed in our hospital includes radiotherapy with standard fractionation (1 fraction per day, 200 cGy per fraction, 5 days per week) according to the National Comprehensive Cancer Network (NCCN) Clinical Practice Guidelines in Oncology (NCCN Guidelines) for Head and Neck Cancer. The planned total dose of radiation therapy was $>60 \mathrm{~Gy}(2.0 \mathrm{~Gy} /$ day $)$ to the primary tumour region and involved nodal stations. In this review, the radiation dose for the primary tumour was defined as the total dose to the primary tumour region (primary tumour site or postoperative tumour bed), and the dose for the neck metastases was defined as the total dose to the involved nodal stations.

Radiotherapy was combined with cisplatin-based triweekly chemotherapy or weekly targeted therapy with cetuximab in this study. The National Health Insurance in Taiwan covers targeted therapy with cetuximab for the patients aged [?]70 years, those with serum creatinine clearance $<50 \mathrm{~mL} / \mathrm{min}$ or hearing impairment.

After treatment completion, regular follow-up was performed once a month in the first year, once every 2 to 3 months thereafter. The first imaging follow-up with computed tomography or magnetic resonance imaging was 3 months after the completion of chemoradiotherapy.

\section{Main outcome measures:}

Disease-specific survival (DSS), disease-free survival (DFS), and overall survival (OS) were calculated using the Kaplan-Meier product limit method. OS was defined as time between the date of treatment initiation and the end of follow-up or death from any cause. DSS was defined as the duration from the date of treatment initiation to the time of death from cancer or treatment-related events. DFS was defined as the duration between treatment completion and date of detection of recurrence. Follow-up was defined as the duration between treatment initiation and last contact. Functional results were determined by dependence on feeding and tracheostomy tubes at last follow-up.

The chi-square test was used to analyse categorical data. The independent $t$-test was used for continuous variables. Kaplan-Meier estimates with the log-rank test were used to evaluate recurrence and survival of patients over time. All statistical tests were performed using SPSS Statistics 25 (IBM, Armonk, NY, USA). A two-sided $p$-value $<0.05$ indicated statistical significance.

\section{Results:}




\section{Patient demographics}

Of the 67 patients reviewed, most were men (95.5\%) with a mean age of 56.1 years. Most patients smoked cigarettes $(90.1 \%)$ and consumed alcohol $(70.2 \%)$. Approximately half had the habit of betel-quid chewing $(50.7 \%)$.

Forty-four patients received primary chemoradiotherapy (CCRT group), and 23 patients received upfront ND followed by chemoradiotherapy (ND + CCRT group). No statistically significant differences were found in patient demographic data or personal habits between the two groups (Table 1).

Cisplatin-based chemotherapy was administered to most patients $(53 / 67,79.1 \%)$, and targeted therapy with cetuximab was administered to 12 patients (17.9\%). Two patients $(3 \%)$ received a combination of targeted therapy and cisplatin-based chemotherapy (Table 2). The ND + CCRT group tended to have earlier T and $\mathrm{N}$ stages than the CCRT group; however, the mean of the maximal size of the neck metastases was similar between the two groups (Table 1). In addition, doses of radiation for primary and neck diseases were lower in the ND + CCRT group with a mean difference of 345.9 cGy for the primary disease $(95 \%$ confidence interval: $173.1,518.8)$ and $562.6 \mathrm{cGy}$ for neck metastases $(95 \%$ confidence interval: $307,818.8)(p=0.000)$ (Table 2).

\section{Type of $N D$}

In the ND + CCRT group, ipsilateral NDs were performed in 21 patients and bilateral NDs in 2 patients. The extent of ND was determined according to a preoperative imaging study and the conclusion of a multidisciplinary meeting.

In the ND + CCRT group, more than half of the patients $(\mathrm{n}=12,52.2 \%)$ had an upstaged pathologic $\mathrm{N}$ stage after ND, compared with the original clinical N stage. The pathological reports of $9(39.1 \%)$ of the 23 patients revealed extranodal extension (ENE).

Recurrence and oncologic outcomes

In all 67 patients, there was no residual neck disease observed in the imaging study performed 3 months after completion of chemoradiotherapy. During the follow-up period, isolated neck recurrence developed in nine patients $(13.4 \%)$, and distant metastasis developed in eight patients $(11.9 \%)$. One patient had both neck and distant metastases (1.5\%). The 3-year DSS, DFS, and OS rates for the whole population were $77.9 \%$, $72.5 \%$, and $68.1 \%$, respectively.

A significantly lower 3-year neck recurrence rate was observed in the ND + CCRT group than in the CCRT group ( $0 \%$ vs. $19 \%, p=0.031$ ). We found no significant difference in the 3 -year distant recurrence rate ( $18.4 \%$ and $10.4 \%, p=0.481)$ or 3 -year DSS $(77.3 \%$ and $75.3 \%, p=0.968)$ between the ND + CCRT and CCRT groups. In addition, there was no significant difference in the 3 -year DFS $(77.3 \%$ and $70.1 \%, p=0.457)$ or 3-year OS (62\% and $71.5 \%, p=0.771)$ between ND + CCRT and CCRT groups (Fig. 1).

\section{Functional outcomes}

At the most recent follow-up, 14 patients $(20.9 \%)$ required tube feeding and $5(7.5 \%)$ required tracheostomy. The ND + CCRT group demonstrated a lower rate of long-term feeding tube dependency $(\mathrm{n}=3,13 \%)$ than the CCRT group $(\mathrm{n}=11,25 \%)$, but the difference was statistically insignificant $(p=0.207)$. Moreover, the rate of long-term tracheostomy dependency was also lower in the ND + CCRT group $(\mathrm{n}=1,4.3 \%)$ than in the CCRT group $(\mathrm{n}=4,9.1 \%)$ but without statistical significance $(p=0.436)$ (Table 2$)$.

Upfront ND in neck lymph node metastases [?]3 $\mathrm{cm}$

The mean maximal size of neck lymph node metastases in the whole population was $3 \mathrm{~cm} ; 31$ patients had a maximal neck metastases [?]3 $\mathrm{cm}$. Compared with those with smaller ones, patients with neck metastases $[?] 3 \mathrm{~cm}$ had a higher rate of 3 -year neck recurrence $(5.8 \%$ and $20.3 \%$, respectively, $p=0.068)$ and 3-year distant recurrence $(3.1 \%$ vs. $26.9 \%, p=0.008)$. Patients with larger neck metastases also had significantly 
poorer outcomes in terms of DSS $(84.8 \%$ vs. $65.9 \%, p=0.03)$ and 3 -year DFS $(84.2 \%$ vs. $59.1 \%, p=0.013)$ but not OS $(77.3 \%$ and $58.1 \%, p=0.222)$.

Eleven patients in the ND + CCRT group and 20 in the CCRT group had maximal nodal size [?] $3 \mathrm{~cm}$. In these patients, the ND + CCRT group had a significantly lower 3-year neck recurrence rate than the CCRT group ( $0 \%$ vs. $32.1 \%, p=0.045)$. However, there was no significant difference in distant metastasis between two groups (28.4\% and 24.5\%, respectively, $p=0.994)$, as well as survival outcomes (Fig. 2).

\section{Upfront $N D$ in early and advanced $N$ stage}

There were 12 patients with early $\mathrm{N}$ stage disease (N1, 3 patients in ND + CCRT group, and 9 patients in CCRT group) and 55 patients with advanced $\mathrm{N}$ stage disease (N2+N3, 20 patients in ND + CCRT group, 35 patients in CCRT group). Among both groups, the ND + CCRT group had a significantly lower 3-year neck recurrence rate than the CCRT group ( $0 \%$ vs. $22.2 \%$ for early disease, $0 \%$ vs. $17.1 \%$ for advanced disease, $p=0.034$ ) (Fig. 3) However, there were no significant differences in distant metastasis and survival outcomes.

\section{Discussion:}

We reviewed a total of 67 patients with p16-negative $\mathrm{cN}+$ OPSCC at our tertiary medical centre. In this series, surgery combined with chemoradiotherapy and primary chemoradiotherapy were both presented as treatment approaches with curative intent. Both modalities showed comparable survival outcomes, while upfront ND followed by chemoradiotherapy allowed for better regional control with significantly reduced radiation dose.

Recently, the treatment of OPSCC has involved a chemoradiotherapeutic organ-preservation approach. ${ }^{(10)}$ However, responses to chemoradiotherapy can vary depending on tumour characteristics, such as p16, N stage, size or volume of the tumour. Among these factors, p16 status is most important. Tumour volume is another significant factor associated with locoregional control and oncological outcome in chemoradiotherapy for head and neck cancers. ${ }^{(11)}$ In the meta-analysis, Xie et al. found that gross tumour volume is a significant factor in evaluating local control and oncological outcomes in patients with head and neck cancers. ${ }^{(12)}$ The response rate to radiotherapy in nodal metastasis appears to be associated with lymph node size, with much lower complete response rates in patients with nodal size [?]3 cm. ${ }^{(13,14)} \mathrm{In}$ our series, huge neck metastases were associated with poorer regional control, distant control, DSS, and DFS but not OS. This finding is consistent with previous studies.

A previous systematic review has evaluated the role of upfront ND in head and neck cancer. ${ }^{(15)}$ However, the result was inconclusive because of high heterogeneity of the analysed data. The management of OPSCC is especially controversial in head and neck cancer due to the nature of $\mathrm{p} 16$, which divides the disease into two distinct diseases. Kelly (2017) conducted a database analysis about upfront surgery in p16(-) OPSCC and showed that both groups had comparable OS. ${ }^{(15)}$ However, they did not provide the information about neck management, neck control benefit and functional outcome. In earlier studies, the authors did not divide patients by p16 status. ${ }^{(16-18)}$ More recent studies focus mostly on p16-positive OPSCCs. A retrospective study in patients with HPV-related OPSCC found that selective ND followed by adjuvant therapy, when indicated, provided excellent long-term regional control. ${ }^{(19)}$

As for p16-negative OPSCC, the role of ND is unclear. Because radiotherapy is less effective in this group with larger neck metastases, ND may play a role. In our study, upfront ND resulted in better regional control, especially in patients with huge neck metastases. Although the effect did not benefit overall survival, it should not be disregarded.

Previous literatures have suggested that ND may negatively impact patients' quality of life due to side effects such as shoulder drop, pain or feeding tube dependence, especially when trimodal treatment was performed. ${ }^{(20-22)}$ However, in our study, long-term feeding dependence and tracheostomy tube dependence were lower in the ND + CCRT group, although not statistically significant. This may have been because most patients $(71 \%)$ in our hospital received selective ND with well-preserved spinal accessory nerve. Further, the 
dose of radiotherapy was also lower in the ND+CCRT group. Common adverse effects of irradiation, such as decreased saliva and damage to pharyngeal constrictors, were related to radiation dose as a continuous variable. The comparable functional outcomes might result from the type of surgery and the dose of radiation.

Another benefit of surgery is that a definite pathological stage can be obtained. Some indicators of poor outcomes, such as ENE and lymph node density, can only be confirmed by ND. In our series, upstaging of nodal disease occurred in half of the patients $(\mathrm{n}=12,52.2 \%)$ after ND. Additionally, microscopic ENE was not detected before the ND in $39.1 \%$ of patients. The presence of ENE is a vital factor on patients' survival, and ND can help stratify patients by definite pathological diagnosis and target or intensify postoperative chemoradiation and post-treatment follow-up for better disease control. In our series, patients with upstaged $\mathrm{N}$ stage after surgery received a higher dose of radiation for neck metastases.

In the future, a larger series of assessments and systematic validations must be considered. Moreover, the role of ND in different subgroups with poorer prognostic factors of the neck, such as ENE and higher lymph node density, must be investigated.

This study has certain limitations including its retrospective design and limited number of cases. The limited number of cases is attributed to the fact that only patients with p16-negative $\mathrm{cN}+$ lesions were enrolled. The $\mathrm{T}$ and $\mathrm{N}$ stages tended to be earlier in the ND + CCRT group than in the CCRT group, which might be explained by the surgeons' selections. ND tended to be performed with primary tumour excision. Thus, ND would be performed more frequently in operable tumours.

\section{Conclusions:}

In this retrospective review, we found that upfront ND reduces regional recurrence in patients with p16negative $\mathrm{cN}+\mathrm{OPSCC}$, and that the effect is more significant in patients with larger neck lymph node metastases. Furthermore, the procedure could achieve comparable oncological outcomes with significantly reduced doses of radiotherapy and obtain a definite pathologic diagnosis. With a reduction in the incidence of p16-positive OPSCC owing to the development of the HPV vaccine, the issue of p16-negative OPSCC and its treatment will be more significant in the future. These findings can help devise a suitable treatment plan when encountering p16-negative OPSCC and highlight the difference in treatment for p16-negative and p16-positive OPSCC.

\section{Data Sharing and Data Availability:}

The data that support the findings of this study are available on request from the corresponding author. The data are not publicly available due to privacy or ethical restrictions.

\section{References:}

1. Chen T-C, Wu C-T, Ko J-Y, Yang T-L, Lou P-J, Wang C-P, et al. Clinical characteristics and treatment outcome of oropharyngeal squamous cell carcinoma in an endemic betel quid region. Scientific Reports. 2020;10(1):526.

2. Reuschenbach M, Tinhofer I, Wittekindt C, Wagner S, Klussmann JP. A systematic review of the HPV-attributable fraction of oropharyngeal squamous cell carcinomas in Germany. Cancer Medicine. 2019;8(4):1908-18.

3. Windon MJ, D'Souza G, Rettig EM, Westra WH, van Zante A, Wang SJ, et al. Increasing prevalence of human papillomavirus-positive oropharyngeal cancers among older adults. 2018;124(14):2993-9.

4. Lassen P, Primdahl H, Johansen J, Kristensen CA, Andersen E, Andersen LJ, et al. Impact of HPVassociated p16-expression on radiotherapy outcome in advanced oropharynx and non-oropharynx cancer. Radiotherapy and Oncology. 2014;113(3):310-6.

5. Kelly JR, Park HS, An Y, Contessa JN, Yarbrough WG, Burtness BA, et al. Comparison of Survival Outcomes Among Human Papillomavirus-Negative cT1-2 N1-2b Patients With Oropharyngeal Squamous 
Cell Cancer Treated With Upfront Surgery vs Definitive Chemoradiation Therapy: An Observational Study. JAMA Oncology. 2017;3(8):1107-11.

6. Pedro C, Mira B, Silva P, Netto E, Pocinho R, Mota A, et al. Surgery vs. primary radiotherapy in early-stage oropharyngeal cancer. Clin Transl Radiat Oncol. 2017;9:18-22.

7. Kelly JR, Park HS, An Y, Yarbrough WG, Contessa JN, Decker R, et al. Upfront surgery versus definitive chemoradiotherapy in patients with human Papillomavirus-associated oropharyngeal squamous cell cancer. Oral oncology. 2018;79:64-70.

8. Golusiński W, Golusińska-Kardach E. Current Role of Surgery in the Management of Oropharyngeal Cancer. Front Oncol. 2019;9:388-.

9. Shama M, Dahl M, Danan D, Silver N, Dziegielewski P. Upfront Surgery Has A Better Outcome in Oropharyngeal cancer HPV- Population2019.

10. Chen AY, Zhu J, Fedewa S. Temporal trends in oropharyngeal cancer treatment and survival: 1998-2009. The Laryngoscope. 2014;124(1):131-8.

11. Dejaco D, Steinbichler T, Schartinger VH, Fischer N, Anegg M, Dudas J, et al. Prognostic value of tumor volume in patients with head and neck squamous cell carcinoma treated with primary surgery. Head \& neck. 2018;40(4):728-39.

12. Xie K, Chen J, Zou J, Chen L, Gong L. Tumor volumes predict prognosis in head and neck cancer: a meta-analysis. Translational Cancer Research. 2017;6(4):687-97.

13. O'Hara J, Simo R, McQueen A, Andi K, Lester S, Giddings C, et al. Management of metastatic neck disease - summary of the 11th Evidence Based Management Day. Clinical Otolaryngology. 2014;39(1):3-5.

14. Paleri V, Urbano TG, Mehanna H, Repanos C, Lancaster J, Roques T, et al. Management of neck metastases in head and neck cancer: United Kingdom National Multidisciplinary Guidelines. J Laryngol Otol. 2016;130(S2):S161-S9.

15. Kelly JR, Park HS, An Y, Contessa JN, Yarbrough WG, Burtness BA, et al. Comparison of Survival Outcomes Among Human Papillomavirus-Negative cT1-2 N1-2b Patients With Oropharyngeal Squamous Cell Cancer Treated With Upfront Surgery vs Definitive Chemoradiation Therapy: An Observational Study. JAMA Oncology. 2017;3(8):1107-11.

16. Sakashita T, Homma A, Hayashi R, Kawabata K, Yoshino K, Iwae S, et al. The role of initial neck dissection for patients with node-positive oropharyngeal squamous cell carcinomas. Oral oncology. 2014;50(7):65761.

17. Moore KMVAaEJ. Focus Issue: Neck Dissection for Oropharyngeal Squamous Cell Carcinoma. International Scholarly Research Notices. 2012;2012.

18. Paximadis PA, Christensen ME, Dyson G, Kamdar DP, Sukari A, Lin HS, et al. Up-front neck dissection followed by concurrent chemoradiation in patients with regionally advanced head and neck cancer. Head \& neck. 2012;34(12):1798-803.

19. Zenga J, Jackson RS, Graboyes EM, Sinha P, Lindberg M, Martin EJ, et al. Oncologic outcomes of selective neck dissection in HPV-related oropharyngeal squamous cell carcinoma. The Laryngoscope. 2017;127(3):623-30.

20. Laverick S, Lowe D, Brown JS, Vaughan ED, Rogers SN. The Impact of Neck Dissection on HealthRelated Quality of Life. Archives of Otolaryngology-Head \& Neck Surgery. 2004;130(2):149-54.

21. Donatelli-Lassig AA, Duffy SA, Fowler KE, Ronis DL, Chepeha DB, Terrell JE. The effect of neck dissection on quality of life after chemoradiation. Otolaryngology-head and neck surgery : official journal of American Academy of Otolaryngology-Head and Neck Surgery. 2008;139(4):511-8. 
22. Lango MN, Egleston B, Ende K, Feigenberg S, D'Ambrosio DJ, Cohen RB, et al. Impact of neck dissection on long-term feeding tube dependence in patients with head and neck cancer treated with primary radiation or chemoradiation. Head \& neck. 2010;32(3):341-7.

23. Gary L. Clayman D, MD; Chad Jeffery Johnson II; William Morrison, MD; et al. The Role of Neck Dissection After Chemoradiotherapy for Oropharyngeal Cancer With Advanced Nodal Disease. JAMA Otolaryngology-Head\&Neck Surgery. 2001;127:5.

24. Goenka A, Morris LG, Rao SS, Wolden SL, Wong RJ, Kraus DH, et al. Long-term regional control in the observed neck following definitive chemoradiation for node-positive oropharyngeal squamous cell cancer. International journal of cancer. 2013;133(5):1214-21.

Table 1. Patient demographics and tumour-related characteristics

\begin{tabular}{|c|c|c|c|c|c|}
\hline & $\mathrm{ND}+\mathrm{CCRT}$ & $\mathrm{ND}+\mathrm{CCRT}$ & CCRT & CCRT & $p$-value \\
\hline Sex $(M: F)$ & $20: 3$ & $20: 3$ & $44: 0$ & $44: 0$ & $p=0.067$ \\
\hline Age & $56.1 \pm 2.21$ & $56.1 \pm 2.21$ & $56.1 \pm 11.1$ & $56.1 \pm 11.1$ & $p=0.992$ \\
\hline Alcohol & $\begin{array}{l}\text { Current }+ \text { quit } \\
\text { non }\end{array}$ & $\begin{array}{l}65.2 \% \\
34.8 \%\end{array}$ & $\begin{array}{l}\text { Current }+ \text { quit } \\
\text { non }\end{array}$ & $\begin{array}{l}72.7 \% \\
27.3 \%\end{array}$ & $p=0.051$ \\
\hline Betel-quid chewing & $\begin{array}{l}\text { Current }+ \text { quit } \\
\text { non }\end{array}$ & $\begin{array}{l}39.1 \% \\
60.9 \%\end{array}$ & $\begin{array}{l}\text { Current }+ \text { quit } \\
\text { non }\end{array}$ & $\begin{array}{l}56.8 \% \\
43.2 \%\end{array}$ & $p=0.388$ \\
\hline Smoking & $\begin{array}{l}\text { Current }+ \text { quit } \\
\text { non }\end{array}$ & $\begin{array}{l}87.0 \% \\
13.0 \%\end{array}$ & $\begin{array}{l}\text { Current }+ \text { quit } \\
\text { non }\end{array}$ & $\begin{array}{l}88.6 \% \\
11.4 \%\end{array}$ & $p=0.907$ \\
\hline $\mathrm{T}$ stage & $\begin{array}{l}1 \\
2 \\
3 \\
4 \mathrm{a} \\
4 \mathrm{~b}\end{array}$ & $\begin{array}{l}3 \\
20 \\
0 \\
0 \\
0\end{array}$ & $\begin{array}{l}1 \\
2 \\
3 \\
4 \mathrm{a} \\
4 \mathrm{~b}\end{array}$ & $\begin{array}{l}1 \\
17 \\
6 \\
13 \\
7\end{array}$ & $p=0.000$ \\
\hline N stage & $\begin{array}{l}1 \\
2 \mathrm{a} \\
2 \mathrm{~b} \\
2 \mathrm{c} \\
3 \mathrm{a} \\
3 \mathrm{~b}\end{array}$ & $\begin{array}{l}7 \\
3 \\
10 \\
1 \\
2 \\
0\end{array}$ & $\begin{array}{l}1 \\
2 \mathrm{a} \\
2 \mathrm{~b} \\
2 \mathrm{c} \\
3 \mathrm{a} \\
3 \mathrm{~b}\end{array}$ & $\begin{array}{l}5 \\
5 \\
15 \\
14 \\
1 \\
4\end{array}$ & $p=0.013$ \\
\hline Mean of max size of neck metastases (mm) & 31.2 & 31.2 & 29.4 & 29.4 & $p=0.660$ \\
\hline
\end{tabular}

ND + CCRT, neck dissection followed by chemoradiotherapy; CCRT, primary chemoradiotherapy; M, male; $\mathrm{F}$, female

Table 2. Radiation dose, chemotherapy regimen, and long-term tube feeding and tracheostomy in the ND + CCRT and CCRT groups

\begin{tabular}{llll}
\hline & ND + CCRT & ND+CCRT & CCRT \\
\hline RT dose of primary tumour (cGy) & $6605.2 \pm 369.4$ & $6605.2 \pm 369.4$ & $6951.1 \pm 235.6$ \\
RT dose of neck metastases (cGy) & $6148.3 \pm 105.1$ & $6148.3 \pm 105.1$ & $6710.9 \pm 468.9$ \\
Regimen of chemotherapy & Cisplatin-based regimen & 20 & Cisplatin-based regimen \\
& Cetuximab & 3 & Cetuximab \\
Tube feeding & Combination of cisplatin-based regimen & 0 & Combination of cisplatin-bas \\
Tracheostomy & 3 & 3 & 11 \\
\hline
\end{tabular}


ND + CCRT, neck dissection followed by chemoradiotherapy; CCRT, primary chemoradiotherapy; RT, radiotherapy
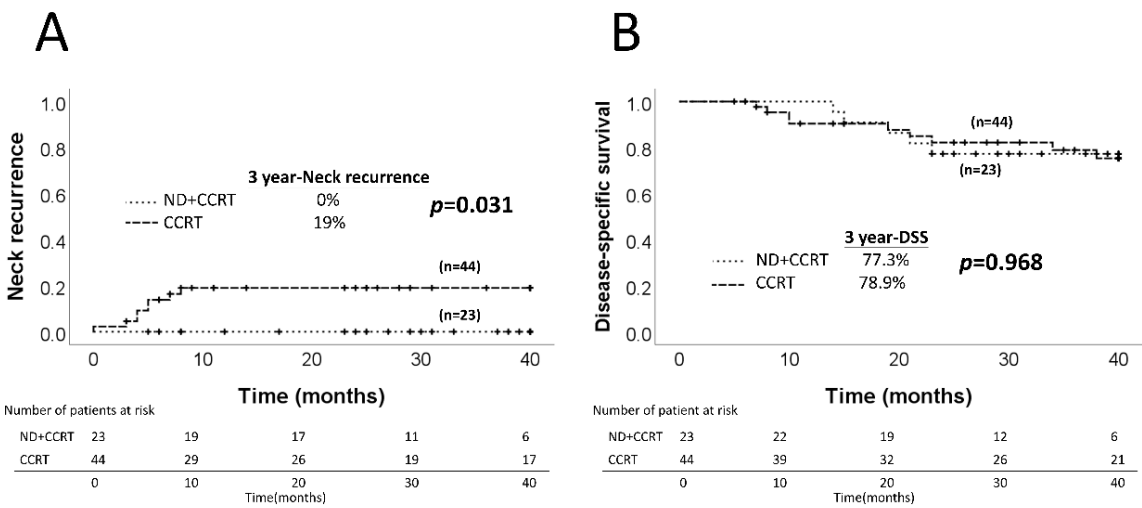

Figure 1 Kaplan-Meier survival curves for neck treatment

Kaplan-Meier survival curve of neck recurrence showing patients who underwent upfront neck dissection had a significantly lower 3-year neck recurrence

Kaplan-Meier survival curves of disease-specific survival showing no significant difference between the two groups

ND + CCRT, neck dissection followed by chemoradiotherapy; CCRT, primary chemoradiotherapy; DSS, disease-specific survival

A

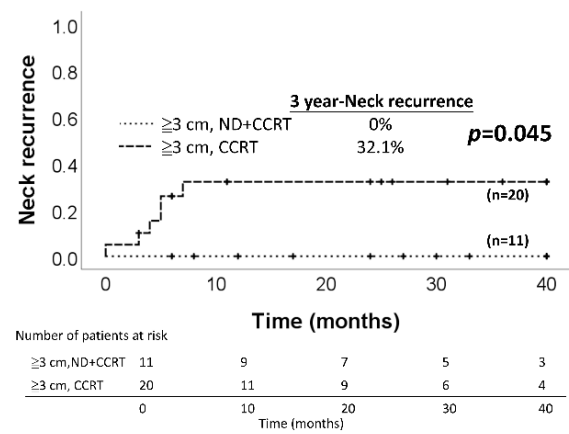

B

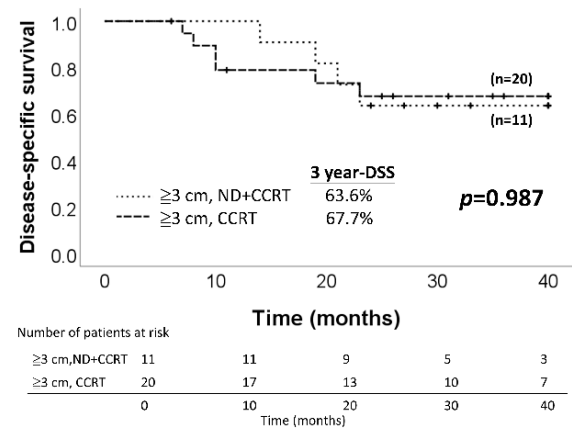

Figure 2 Kaplan- Meier survival curve for neck treatment in patients with neck metastases [?]3 $\mathrm{cm}$

(A) Kaplan-Meier survival curve of neck recurrence showing that patients with neck metastases [?]3 $\mathrm{cm}$ who underwent upfront neck dissection had a significantly lower neck recurrence

(B) Kaplan-Meier survival curve of disease-specific survival showing no significant difference between the two groups

[?]3 cm, ND + CCRT, neck dissection followed by chemoradiotherapy in patients with neck size [?]3 cm; [?]3 $\mathrm{cm}$, CCRT, primary chemoradiotherapy in patients with neck size [?]3 cm; DSS, disease-specific survival 
A

B

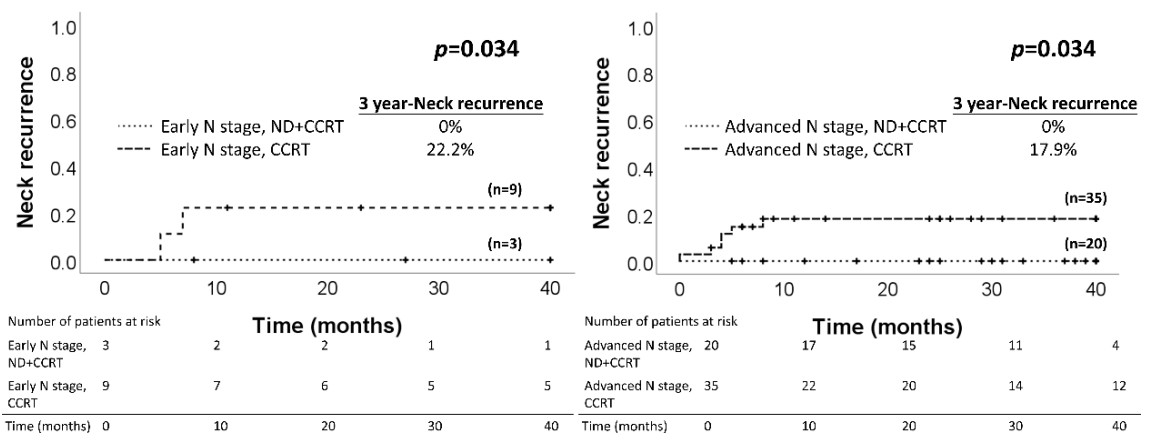

Figure 3 Kaplan- Meier survival curve for neck treatment in patients with early N stage disease or advanced $\mathrm{N}$ stage disease

(A) Kaplan-Meier survival curve of neck recurrence showing that patients with early $\mathrm{N}$ stage disease who underwent upfront neck dissection had a significantly lower neck recurrence

(B) Kaplan-Meier survival curve of neck recurrence showing that patients with advanced $\mathrm{N}$ stage disease who underwent upfront neck dissection had a significantly lower neck recurrence

Early N stage, ND + CCRT, neck dissection followed by chemoradiotherapy in patients with early N stage; Early N stage, CCRT, primary chemoradiotherapy in patients with early N stage; Advanced N stage, ND + CCRT, neck dissection followed by chemoradiotherapy in patients with advanced N stage; Advanced N stage, CCRT, primary chemoradiotherapy in patients with advanced $\mathrm{N}$ stage

\section{Hosted file}

Table 1.pdf available at https://authorea.com/users/402952/articles/514656-upfrontneck-dissection-in-chemoradiotherapy-for-p16-negative-oropharyngeal-cancer-with-neckmetastases-a-retrospective-study

\section{Hosted file}

Table 2.pdf available at https://authorea.com/users/402952/articles/514656-upfrontneck-dissection-in-chemoradiotherapy-for-p16-negative-oropharyngeal-cancer-with-neckmetastases-a-retrospective-study
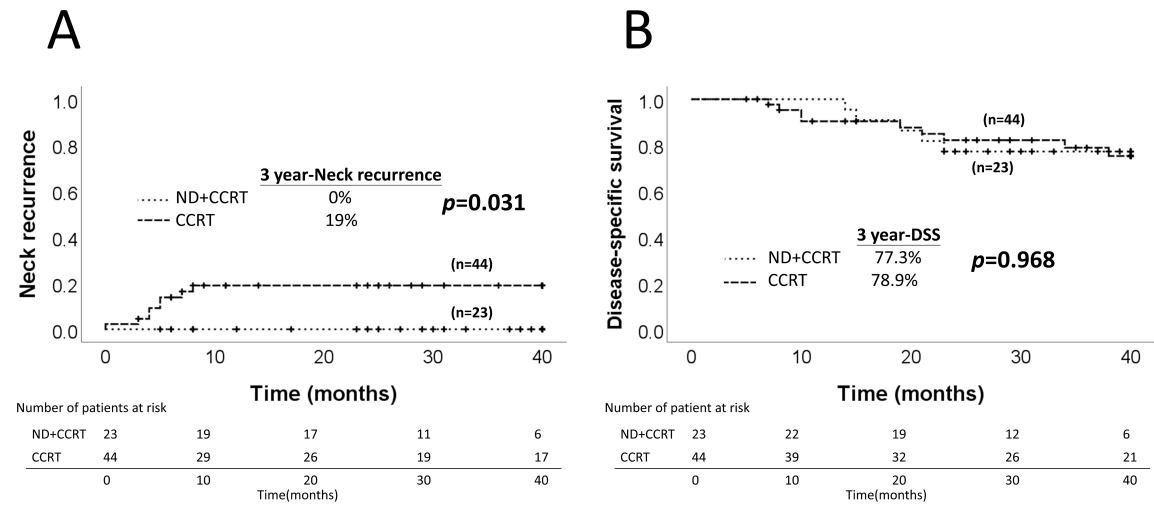
A

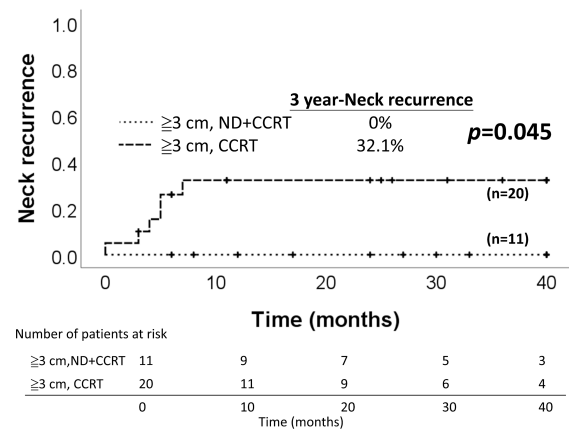

A

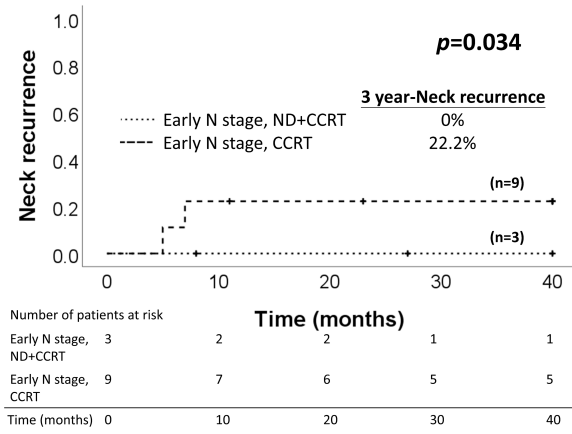

B

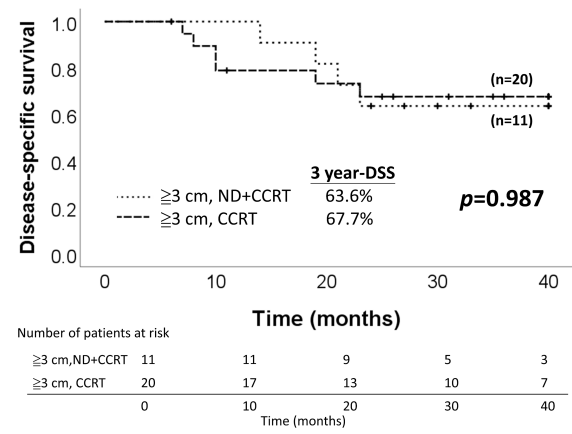

B

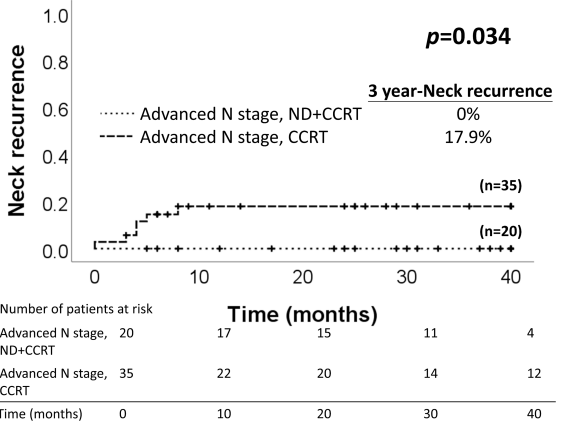

